successive columns give the plate number, the date, the length of exposure, the Julian Day, and the magnitude. On Plate AM 4 I 43, there are 17 exposures of from 25 to 30 minutes each.

Table I. Observations of the Nova.

\begin{tabular}{|c|c|c|c|c|c|c|c|c|c|c|c|}
\hline Plate & Date & Exp. & J. D. & Magn. & Plate & & Date & & Exp. & J. D. & Magn. \\
\hline $\mathrm{AM}_{3718}$ & 1905 July I2 & $60^{m}$ & $7 \circ 39$ & $<$ II. 5 & $\mathrm{AM}_{4185}$ & 1906 & April & 28 & $60^{m}$ & $73^{29}$ & 10.55 \\
\hline $\mathrm{AM}_{4015}$ & Dec. 5 & 62 & 7185 & $9.7^{2}$ & $\mathrm{AM}_{4194}$ & 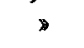 & $\Rightarrow$ & 30 & 60 & 7331 & 10.42 \\
\hline $\mathrm{AM}_{4053}$ & I 906 Jan. 26 & 60 & 7237 & 9.72 & $\mathrm{AM}_{4212}$ & 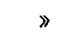 & May & Io & 60 & 7341 & I0.10 \\
\hline $\mathrm{AM}_{4076}$ & $\Rightarrow \quad 30$ & 63 & 7241 & 9.98 & $\mathrm{AM}_{4225}$ & $\gg$ & $>$ & 12 & 60 & 7343 & IO.I 5 \\
\hline $\mathrm{AM}_{409} 8$ & Febr. 3 & 60 & 7245 & 9.95 & $\mathrm{AM}_{4287}$ & 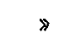 & June & I I & 64 & 7373 & 10.02 \\
\hline $\mathrm{AM}_{4} \mathrm{I} 33$ & Mar. I 7 & 75 & 7287 & 9.80 & $\mathrm{AM} 4300$ & 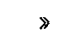 & $\gg$ & I 3 & 65 & 7375 & 9.80 \\
\hline $\mathrm{AM}_{4134}$ & $\gg \quad 21$ & 70 & 7291 & 9.78 & $\mathrm{AM}_{435^{2}}$ & $\searrow$ & $\gg$ & 29 & 60 & 7391 & 11.05 \\
\hline $\mathrm{AM}_{4} \mathrm{I} 43$ & 26 & 485 & 7296 & 9.80 & $\mathrm{AM}_{43} 64$ & 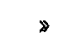 & July & 2 & 60 & 7394 & < II.2 \\
\hline
\end{tabular}

The number of early plates examined, on which the Nova was not seen and was fainter than the eleventh magnitude, was 127 , the numbers taken in successive years being as follows: in 1889 one, 1890 two, $189 \mathrm{I}$ one, 1892 one, I 893 three, 1894 eight, 1895 eleven, 1896 ten, 1897 eighteen, I 898 eleven, 1899 six, I 900 eleven, I 901 ten, 1902 eleven, 1902 seven, 1904 nine, I 905 seven. Two of these plates taken with the 24 -inch Bruce Telescope, and two taken with the 8 -inch Bache Telescope, show stars at least as

faint as the magnitude 15.0 , and twentyfive other plates show stars as faint as the magnitude 13.5 , but no trace of the Nova could be found on any of them.

The Nova shows considerable fluctuation in light during the period covered by the observations, and it is not impossible that it may again become sufficiently bright for its spectrum to be obtained. Even without such proof, there can be little doubt that the object observed is actually a nova.

Harvard College Observatory, 1906 Nov. I 2.

Edward C. Pickering.

\title{
Observation d'une blanche tache sur le III. satellite de Jupiter.
}

Je me fais un devoir d'appeler tout de suite l'attention des astronomes possédant des puissants instruments, sur la très blanche tache polaire boréale que j'ai observé hier

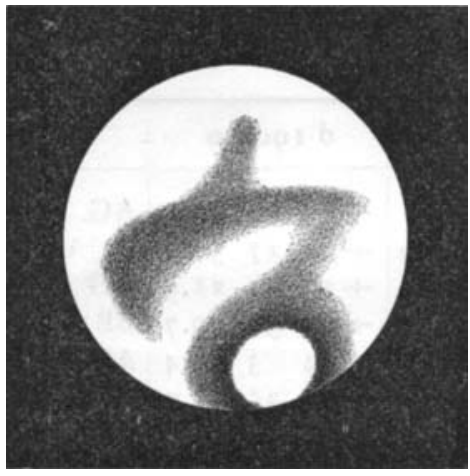

Satellite III de Jupiter. 1906 Nov. $2313^{\mathrm{h}} 30^{\mathrm{m}}$.

Barcelone, le 24 novembre igo6.
23 novembre 1906, sur le troisième satellite de Jupiter avec l'équatorial de $38 \mathrm{~cm}$ de l'Observatoire Fabra.

Elle est d'une éclatante blancheur, bordée d'une région très sombre, à peu près comme on observe dans la planète Mars, et d'une observation relativement facile. Je l'ai observé avec des grossissements de $45^{\circ}$ à $75^{\circ}$ fois. Avec ce dernier grossissement, l'observation de cette tache était d'une facilité extraordinaire. Je crois qu'elle peut être visible avec des instruments bien plus petits.

Le diamètre du satelite passant par le centre de la tache était sensiblement perpendiculaire à la direction des bandes de Jupiter, mais la tache paraissait penchée vers nous. Cet observation est faite entre $13^{\mathrm{h}}$ et $14^{\mathrm{h}}$ I $5^{\mathrm{m}}$. Pendant ce temps, je n'ai observé aucun déplacement de la tache brillante.

On voyait aussi, évidemment, des autres taches sombres dans le reste du disque, mais il etait très difficile de les voir avec quelque précision. L'image télescopique était très belle. Dans son ensemble, le satellite III paraissait une image réduite de la planète Mars.

Fosé Comas Solá, Directeur de l'Observatoire Fabra.

\section{Beobachtungen von kleinen Planeten.}

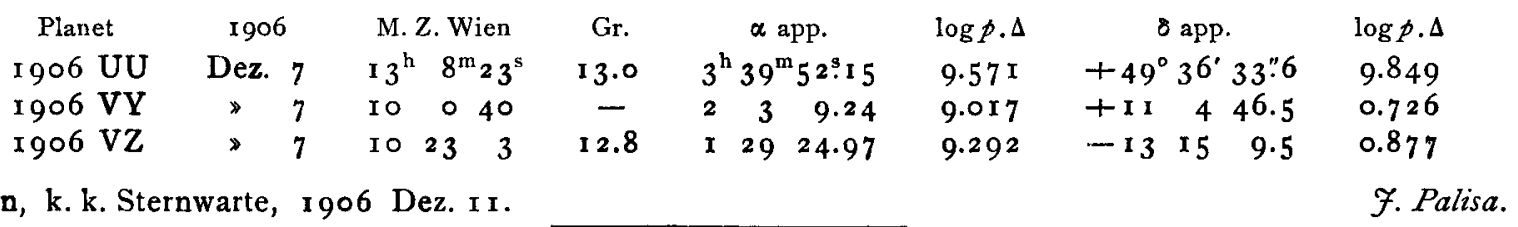

